\title{
The protective effect of thymoquinone over olanzapine- induced side effects in liver, and metabolic side effects
}

\author{
Bilgic $\mathrm{S}^{1}$, Tastemir Korkmaz D ${ }^{1}$, Azirak $\mathrm{S}^{1}$, Guvenc $\mathrm{AN}^{1}$, Kocaman $\mathrm{N}^{2}$, Ozer $\mathrm{MK}^{3}$ \\ University of Adiyaman, Vocational School of Health Services, Adiyaman, Turkey. \\ sbilgic@adiyaman.edu.tr
}

\begin{abstract}
OBJECTIVES: The aim of the study was to investigate the possible protective qualities of thymoquinone (TQ) against the side-effects of olanzapine (OLZ) in an experimental model in rat liver with histologic and biochemical assessments.

METHODS: Experimental procedures were performed on 35 female Sprague Dawley rats. Rats were randomly divided into five groups as: group 1: control; group 2: OLZ; group 3: OLZ+TQ-1; group 4: OLZ+TQ-2; and group 5: OLZ+TQ-3.

RESULTS: The results showed that a 2-week administration of OLZ (4 mg/kg, once a day for the first week, $8 \mathrm{mg} / \mathrm{kg}$ once a day for the second week, p.o.) and treatment with TQ (25, 50, $100 \mathrm{mg} / \mathrm{kg}$, once daily, p.o.) significantly reduced weight gain induced by OLZ. In addition, TQ increased the total antioxidant status (TAS), high-density lipoprotein cholesterol (HDL), insulin levels and decreased serum oxidative stress index (OSI), total oxidant status (TOS), alanine aminotransferase (ALT), aspartate aminotransferase (AST), gamma glutamyl transpeptidase (GGT), low density lipoprotein cholesterol (LDL), glucose, triglycerides (TG) and total cholesterol $(\mathrm{CH})$ levels significantly $(p<0.05)$.

CONCLUSION: This study revealed that treatment with TQ might protect liver tissue against the side-effects of OLZ. TQ could be an effective course of therapy to enhance therapeutic efficacy (Tab. 4, Fig. 4, Ref. 47). Text in PDF www.elis.sk.

KEY WORDS: thymoquinone, olanzapine, adverse effects, liver, weight gain/loss, apoptosis.
\end{abstract}

\begin{abstract}
Abbreviations: ALT - alanine aminotransferase, AST - aspartate aminotransferase, $\mathrm{CH}$ - total cholesterol, DAB - diaminobenzidine, GGT - gamma glutamyl transpeptidase, HDL-cholesterol high-density lipoprotein cholesterol, LDL-cholesterol - low-density lipoprotein cholesterol, OLZ - olanzapine, OSI - oxidative stress index, PBS - phosphate buffered saline, ROS - reactive oxygen species, TAS - serum total antioxidant status, TG - triglycerides, TOS - total oxidant status, TQ - thymoquinone, TUNEL - terminal deoxynucleotidyl transferase dUTP nick-end labeling.
\end{abstract}

\section{Introduction}

OLZ is one of the atypical antipsychotics, drugs widely used in the treatment of psychotic disorders that have also been used as monotherapy or adjunctively with antidepressants to treat depressive disorders with or without psychotic symptoms (1). Clinical assay suggests that OLZ reduces both positive and negative symptoms, and that it is associated with a low incidence of extra pyramidal side effects. In addition, it is related to significant weight

${ }^{1}$ University of Adiyaman, Vocational School of Health Services, Adiyaman, Turkey, ${ }^{2}$ University of Firat, Department of Histology, Faculty of Medicine, Elaziğ, Turkey, and ${ }^{3}$ University of Adiyaman, Department of Pharmacology, Faculty of Medicine, Adiyaman, Turkey

Address for correspondence: S. Bilgiç, University of Adiyaman, Vocational School of Health Services, TR02040 Altinşehir-Adiyaman, Turkey. Phone: +90.4162233800-4181, Fax: +90.4162232071 gain and metabolic alterations such as dyslipidemias, diabetes, and other problems involving the metabolic syndrome $(2,3)$. There are few cases declaring OLZ associated hepatotoxicity in patients, but the mechanism that leads to OLZ-related liver toxicity is not well known (4). The primary site for OLZ metabolism is the liver, where it is metabolized into inactive metabolites primarily by the cytochrome P450 system. However, although treatments with atypical antipsychotics are effective in stress-associated psychiatric diseases like schizophrenia and mood and anxiety disorders, the influence of OLZ on the liver requires further research (5).

Medicinal plants nowadays are an important source of drug synthesis. The wide utilization of herbal drugs has encouraged scientists to research their impressive effects on health, and a large number of medicinal plants and their active extracted ingredients are extensively studied for their potentials to protect cells from injuries. Nigella sativa (also called black cumin, black seed) is among the promising medicinal plants. $N$. sativa Linn as a member of the botanical family of Ranunculaceae is an annual herbaceous plant (6). TQ (2-isopropyl-5-methyl-1,4-benzoquinone), the main active component of the essential oil of Nigella sativa seeds, has various pharmacological effects. It has been reported to display various pharmacological activities such as antioxidant (7), antidiabetic (8), cardioprotective (9), hepatoprotective (10), neuroprotective (11), nephroprotective (12), anti-inflammatory (13), anti-mutagenic (14), and anticancer (15) effects. TQ is of low toxicity (LD50 2.4 $\mathrm{g} / \mathrm{kg}$ ) and is well tolerated when given subchronically till $90 \mathrm{mg} /$ 
$\mathrm{kg}$ /day for 90 days (16). TQ supplementation considerably protected several organs including liver, against oxidative damage induced by a variety of free radical generating agents (17). The high potency and low systemic toxicity of TQ make it a promising alternative to conventional therapeutic drugs (18). The fact that OLZ exposure induces an excessive increase in metabolic alterations suggests that TQ could be used as an alternative therapy.

To our knowledge, there is no report regarding the protective and therapeutic effects of TQ against OLZ-induced liver toxicity. The influence of TQ on OLZ-induced liver injury has not been studied until now. Therefore, the present study was designed to investigate, for the first time, the possible beneficial impact of oral supplementation with TQ against OLZ-induced liver damage in rats. To achieve our goal, we performed several biochemical and histological analyses in female rats.

\section{Materials and methods}

\section{Chemicals}

OLZ was obtained from Ali Arif Ilac Sanayi (ARIS), Istanbul, Turkey. TQ (purity $>98 \%$ ) was purchased from Sigma. All other chemicals used were of the best analytical grade.

\section{Animals}

In this study, 35 female Sprague Dawley rats were used. Healthy adult (4 months old) rats, weighing 250-300 g obtained from the Firat University Laboratory Animal Production and Research Center. Entire animal care and follow-up were performed at this Center. The experiments were performed in accordance with the protocol approved by Firat University Faculty of Medicine, Laboratory Animals Ethics Committee (Protocol \# 2015/23). The rats were kept at $21 \pm 1^{\circ} \mathrm{C}$, for $12 \mathrm{~h}$ in a light-dark cycle, and were fed with standard rat chow and drunk tap water ad libitum. OLZ and TQ were administered to rats for 2 weeks.

\section{Experimental design}

In our study, 35 rats were randomly assigned to one of five groups, with an equal number of rats contained within each group. Simple randomization technique was used in this experimental study. Thirty-five female Sprague Dawley rats were divided into five groups as follows: group 1: control; group 2: OLZ; group 3: OLZ+TQ-1; group 4: OLZ+TQ-2; group 5: OLZ+TQ-3. The control group was given physiological saline solution by gavage once a day. Except for control, OLZ was given to all groups in amount of $4 \mathrm{mg} / \mathrm{kg}$ once a day for the first week, and $8 \mathrm{mg} / \mathrm{kg}$ once a day for the second week. OLZ+TQ-1 group was given TQ in amount of $25 \mathrm{mg} / \mathrm{kg}$ body weight/day. OLZ+TQ-2 group was given TQ in amount of $50 \mathrm{mg} / \mathrm{kg}$ body weight/day. OLZ+TQ-3 group was given TQ in amount of $100 \mathrm{mg} / \mathrm{kg}$ body weight/day. The assigned dosage of powdered OLZ was administered according to a previous report which demonstrated a significant weight gain in female Sprague Dawley rats (19). TQ was administered by gastric tube daily between 8:00 and 9:00 a.m. The dose and duration of TQ was selected according to results from previous study (20). All compounds were suspended in physiological saline solution and administered by gavage once a day. Body weight was recorded at the beginning and at the end of the study. The treatment course lasted 2 weeks for all groups. At the end of week 2 of the treatment period, the animals were euthanized by exsanguination through cardiac puncture under diethyl ether anesthesia. The entire liver was excised and stored at $-80{ }^{\circ} \mathrm{C}$ for subsequent biochemical analysis. Blood samples were immediately collected from the retro-orbital venous plexus in a tube free from any anticoagulant agent for separation of serum samples for biochemical analysis.

\section{Liver enzyme assays}

Blood samples collected from each rat were used to determine liver enzyme activity. Serum concentrations of $\mathrm{CH}(\mathrm{mg} / \mathrm{dL})$, HDLcholesterol $(\mathrm{mg} / \mathrm{dL})$, and TG $(\mathrm{mg} / \mathrm{dl})$ were measured using routine enzymatic methods with Olympus 2700 analyzer (Olympus Diagnostica GmbH, Hamburg, Germany). LDL-cholesterol (mg/dL) levels were calculated using Friedewald's equation. Standard liver function tests, namely ALT (U/L), AST (U/L), GGT (U/L) were measured on the same day with an autoanalyzer. We examined the glucose level (Architect ci16200) and insulin level (Beckman Coulter) according to manufacturer's instructions.

\section{TAS and TOS determination}

The automated calorimetric measurement methods developed by Erel were used to determine TAS ( $\mathrm{mmol} / \mathrm{L})$ and TOS $(\mu \mathrm{mol} / \mathrm{L})$ (a serum oxidant parameter). Measurements of TAS and TOS in serum samples were defined by TAS and TOS kit (REL Assay Diagnostics) $(21,22)$.

\section{OSI determination}

OSI was defined as TOS-to-TAS ratio and calculated as follows: OSI (arbitrary unit $)=((\mathrm{TOS}, \mu \mathrm{mol} \mathrm{H} 2 \mathrm{O} 2 \mathrm{eq} / \mathrm{L}) /(\mathrm{TAS}, \mu \mathrm{mol}$ Trolox eq/L)) (23).

Terminal deoxynucleotidyl transferase dUTP nick-end labeling (TUNEL) assay

Apoptotic cells were defined using the ApopTag Plus Peroxidase In Situ Apoptosis Detection Kit (Chemicon, Cat no: S7101, USA) according to the manufacturer's instructions. Sections (5 $\mu \mathrm{m})$ taken from the paraffin blocks were entrenched onto polylysine-coated slides, deparaffinized using xylene, dehydrated with a series of alcohol rinses, and then washed with phosphate-buffered saline (PBS). The tissues were then incubated with a proteinase K solution $(0.05 \%)$ and with $3 \%$ hydrogen peroxide for $5 \mathrm{~min}$ to forestall endogenous peroxidase activity. After washing with PBS, the tissues were incubated with equilibration buffer for 6 min and in working solution $(70 \% \mu \mathrm{L}$ reaction Buffer $+30 \%$ TdT enzyme) at $37^{\circ} \mathrm{C}$ under moist conditions for $60 \mathrm{~min}$. Tissues were then incubated in stop/wash buffer for $10 \mathrm{~min}$ and incubated in anti-digoxigenin-peroxidase for $30 \mathrm{~min}$. Apoptotic cells were examined using the diaminobenzidine substrate. Cross-sections contrast-stained with methyl green were sealed using a proper covering solution. Mammary tissue was used as a positive control. PBS was used instead of TdT enzyme in the negative control tissue. Preparations were examined and assessed using a research micro- 
618-625

Tab. 1. Body weight (g) of animals during treatment.

\begin{tabular}{|c|c|c|c|c|c|}
\hline Design of treatment & Control & OLZ & OLZ+TQ-1 & OLZ+TQ-2 & OLZ+TQ-3 \\
\hline Start of Study & $258.57 \pm 8.39$ & $240.33 \pm 8.67$ & $255.20 \pm 5.23$ & $260.83 \pm 5.95$ & $250.40 \pm 5.50$ \\
\hline End of Study & $269.14 \pm 8.17$ & $249.50 \pm 9.02$ & $245.80 \pm 3.55$ & $249.16 \pm 9.80$ & $234.20 \pm 7.95$ \\
\hline \multicolumn{6}{|c|}{ Statistical comparison (Start of study vs end of study) (P) } \\
\hline Control & 0.011 & & & & \\
\hline OLZ & 0.001 & & & & \\
\hline OLZ+TQ-1 & 0.015 & & & & \\
\hline OLZ+TQ-2 & 0.312 & & & & \\
\hline OLZ+TQ-3 & 0.127 & & & & \\
\hline
\end{tabular}

Changes in the body weight of experimental rats. Values are expressed as mean \pm SEM of seven animals. The groups were compared with the paired-samples T-test at initial and final of the treatment. $\mathrm{p} \leq 0.05$. OLZ - olanzapine; TQ - thymoquinone; OLZ+TQ-1 - OLZ+25 mg/kg TQ; OLZ+TQ-2 - OLZ+50 mg/kg TQ; OLZ+TQ-3 - OLZ+100 $\mathrm{mg} / \mathrm{kg}$ TQ. Amounts of $4 \mathrm{mg} / \mathrm{kg}$ once a day for the first week, $8 \mathrm{mg} / \mathrm{kg}$ once a day for the second week of OLZ were given to all groups, except for control group

Tab. 2. Comparison of serum oxidative stress parameters among the groups.

\begin{tabular}{lccccc}
\hline Parameters & Control & OLZ & OLZ+TQ-1 & OLZ+TQ-2 & OLZ+TQ-3 \\
\hline TOS $(\mu \mathrm{mol} / \mathrm{L})$ & $9.75 \pm 1.66^{\mathrm{b}, \mathrm{c}}$ & $17.32 \pm 1.99^{\mathrm{a}, \mathrm{c}, \mathrm{d}, \mathrm{e}}$ & $4.91 \pm 0.93^{\mathrm{a}, \mathrm{b}, \mathrm{e}}$ & $7.56 \pm 0.42^{\mathrm{b}, \mathrm{e}}$ & $12.23 \pm 0.66^{\mathrm{b}, \mathrm{c}, \mathrm{d}}$ \\
TAS $(\mathrm{mmol} / \mathrm{L})$ & $1.69 \pm 0.26^{\mathrm{b}, \mathrm{c}, \mathrm{e}}$ & $0.62 \pm 0.11^{\mathrm{a}, \mathrm{c}, \mathrm{d}}$ & $3.55 \pm 0.11^{\mathrm{a}, \mathrm{b}, \mathrm{d}, \mathrm{e}}$ & $1.45 \pm 0.18^{\mathrm{b}, \mathrm{c}, \mathrm{e}}$ & $0.76 \pm 0.13^{\mathrm{a}, \mathrm{c}, \mathrm{d}}$ \\
OSI $(\mathrm{AU})$ & $6.07 \pm 0.82^{\mathrm{b}}$ & $44.01 \pm 17.45^{\mathrm{a}, \mathrm{c}, \mathrm{d}}$ & $1.36 \pm 0.23^{\mathrm{b}}$ & $6.07 \pm 1.17^{\mathrm{b}}$ & $17.83 \pm 2.94$ \\
\hline
\end{tabular}

Each group represents the mean \pm SEM for seven rats. ${ }^{a} \mathrm{p}<0.01$ vs control group; ${ }^{b} \mathrm{p}<0.01$ vs OLZ group; ${ }^{\mathrm{p}}<0.01$ vs OLZ+TQ-1 group; ${ }^{\mathrm{d}} \mathrm{p}<0.03$ vs OLZ+TQ-2 group; ${ }^{\mathrm{e}} \mathrm{p}<0.03$ vs OLZ+TQ-3 group.

OLZ - olanzapine; TQ - thymoquinone; TAS - total antioxidant status; TOS - total oxidant status; OSI - oxidative stress index; OLZ+TQ-1 - OLZ+25 mg/kg TQ; OLZ+TQ-2 - OLZ+50 mg/kg TQ; OLZ+TQ-3 - OLZ+100 mg/kg TQ. Amounts of $4 \mathrm{mg} / \mathrm{kg}$ once a day for the first week, $8 \mathrm{mg} / \mathrm{kg}$ once a day for the second week of OLZ were given to all groups, except for control group. AU - arbitrary units

scope (Olympus BH2 light microscope) and then photographed. To assess the TUNEL staining, after staining with methyl green, cells with green nuclei were considered normal whereas those with brown nuclei were considered apoptotic. Apoptotic (TUNEL-positive) cells were counted and statistically assessed. This analysis was made in at least eight areas of each liver section (two sections/ animal), and the sections were analyzed at $400 \times$ magnification (24).

The evaluation of TUNEL staining was made based on the extent of the staining of apoptotic cells. The extent of TUNEL staining was scored semiquantitatively as 0 (none), 1 (light), 2 (medium), and 3 (intense) (25).

\section{Statistical analysis}

Statistical analyses were performed using a statistical software package (SPSS version 20.0, SPSS, Chicago, IL). Normality for variables in the groups was determined by the Shapiro-Wilk test. All values are given as means \pm standard error of mean (SEM). Body weight gain data were analyzed by paired-samples T-test. The groups were compared with the paired-samples T-test at the beginning of study and end of the study. Biochemical parameters showed a normal distribution. One-way analysis of variance (ANOVA) and post-hoc LSD tests were used to compare the groups. For histopathological analysis, results were expressed as means \pm standard deviation. The statistical significant difference was determined by ANOVA followed by Tukey's multiple comparison test. Probability values (p) less than 0.05 were considered to be statistically significant.

\section{Results}

Body weight measurements showed that during the 2 weeks animals grew from $258.57 \mathrm{~g}$ at day 1 to $268.14 \mathrm{~g}$ for the control

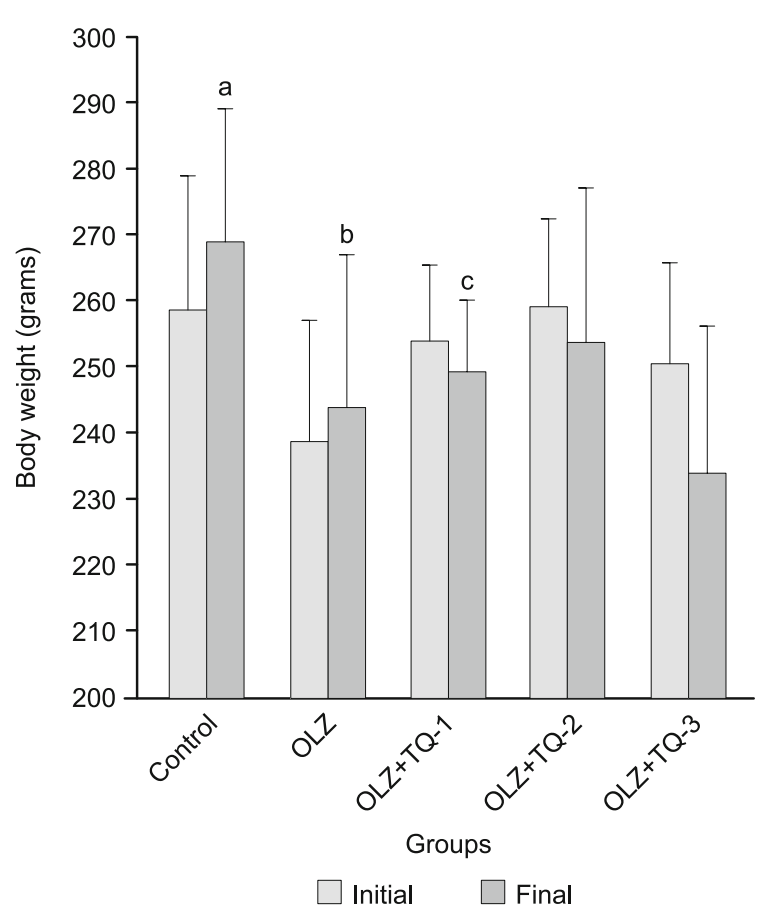

Fig. 1. Changes in the body weight of experimental rats. Values are expressed as mean $\pm \mathrm{SEM}$ of seven animals. The groups were compared with the $p$ aired-samples T-test at the beginning and end of the treatment. a,b,c In each column, different superscript letters mean significant differences at $p<0.05$. OLZ: olanzapine; TQ: thymoquinone; OLZ+TQ-1: OLZ+25 mg/kg TQ; OLZ+TQ-2: OLZ+50 mg/kg TQ; OLZ+TQ-3: OLZ+100 mg/kg TQ. Amounts of $4 \mathrm{mg} / \mathrm{kg}$ once a day for the first week, $8 \mathrm{mg} / \mathrm{kg}$ once a day for the second week of OLZ were given to all groups, except for control group. 

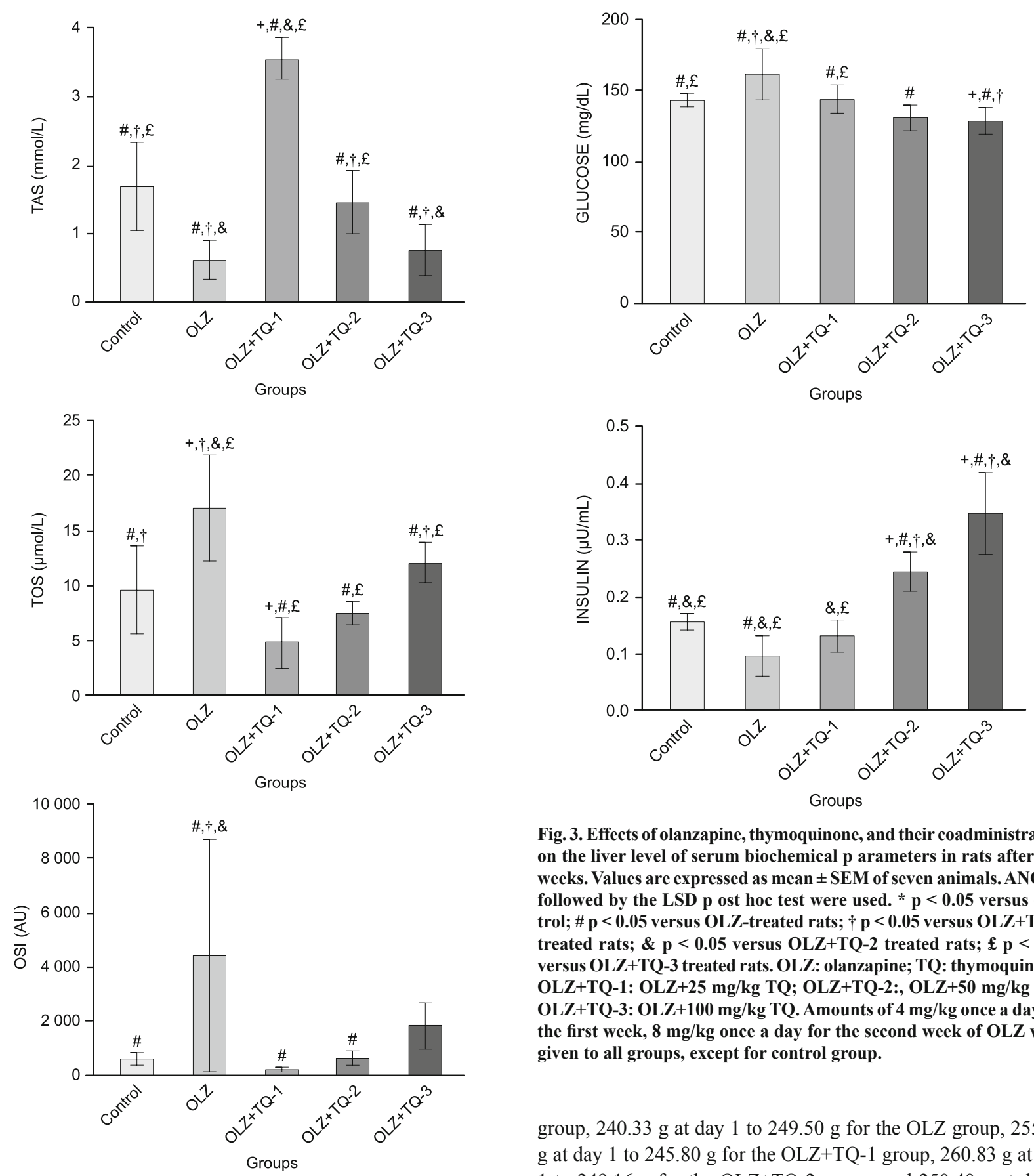

Fig. 2. Effects of olanzapine, thymoquinone, and their coadministration on the liver level of TAS (total antioxidant status), TOS (total oxidant status), and OSI (oxidative stress index) in rats after two weeks. Values are expressed as mean \pm SEM of seven animals. ANOVA followed by the LSD p ost hoc test were used. * $\mathbf{p}<0.05$ versus control; \# $\mathbf{p}<$ 0.05 versus $O L Z$-treated rats; $\uparrow p<0.05$ versus $O L Z+T Q-1$ rats; $\&$ $\mathrm{p}<0.05$ versus $\mathrm{OLZ}+\mathrm{TQ}-2$ rats; $£ \mathrm{p}<0.05$ versus $\mathrm{OLZ}+\mathrm{TQ}-3$ rats. OLZ: olanzapine; TQ: thymoquinone; OLZ+TQ-1: OLZ+25 mg/kg TQ; OLZ+TQ-2: OLZ+50 mg/kg TQ; OLZ+TQ-3: OLZ+100 mg/kg $T Q$. Amounts of $4 \mathrm{mg} / \mathrm{kg}$ once a day for the first week, $8 \mathrm{mg} / \mathrm{kg}$ once a day for the second week of OLZ were given to all groups, except for control group. AU: arbitrary units.

Fig. 3. Effects of olanzapine, thymoquinone, and their coadministration on the liver level of serum biochemical $p$ arameters in rats after two weeks. Values are expressed as mean \pm SEM of seven animals. ANOVA followed by the LSD p ost hoc test were used. * $p<0.05$ versus control; $\# p<0.05$ versus OLZ-treated rats; $\uparrow p<0.05$ versus $O L Z+T Q-1$ treated rats; \& $p<0.05$ versus $O L Z+T Q-2$ treated rats; $£ p<0.05$ versus $O L Z+T Q-3$ treated rats. OLZ: olanzapine; TQ: thymoquinone; OLZ+TQ-1: OLZ+25 mg/kg TQ; OLZ+TQ-2:, OLZ+50 mg/kg TQ; OLZ+TQ-3: OLZ+100 mg/kg TQ. Amounts of $4 \mathrm{mg} / \mathrm{kg}$ once a day for the first week, $8 \mathrm{mg} / \mathrm{kg}$ once a day for the second week of OLZ were given to all groups, except for control group.

group, $240.33 \mathrm{~g}$ at day 1 to $249.50 \mathrm{~g}$ for the OLZ group, 255.20 $\mathrm{g}$ at day 1 to $245.80 \mathrm{~g}$ for the OLZ+TQ-1 group, $260.83 \mathrm{~g}$ at day 1 to $249.16 \mathrm{~g}$ for the OLZ+TQ-2 group, and $250.40 \mathrm{~g}$ at day 1 to $234.20 \mathrm{~g}$ for the OLZ+TQ-3 group at day $14(\mathrm{p}=0.011, \mathrm{p}=$ $0.001, \mathrm{p}=0.015, \mathrm{p}=0.312, \mathrm{p}=0.127$, respectively). There was a significantly increased total body weight gain in the control, OLZ groups and a significantly decreased body weight gain in the OLZ+TQ-1 treatment group $(\mathrm{p}=0.011, \mathrm{p}=0.001, \mathrm{p}=0.015$, respectively). However, the OLZ+TQ-2 and OLZ+TQ-3 groups were observed to have a decreased weight gain and had no significant effect on these measurements $(p=0.312, p=0.127$, respectively) (Tab. 1, Fig. 1, paired-samples T-test for the body weight at day 14). 
Tab. 3. Levels of serum biochemical parameters for all groups.

\begin{tabular}{|c|c|c|c|c|c|c|}
\hline Parameters & Control & OLZ & OLZ+TQ-1 & OLZ+TQ-2 & OLZ+TQ-3 & $\mathrm{p}$ \\
\hline ALT (U/L) & $64.00 \pm 1.77^{\mathrm{b}, \mathrm{d}, \mathrm{e}}$ & $74.28 \pm 0.99^{\mathrm{a}, \mathrm{c}, \mathrm{d}, \mathrm{e}}$ & $67.33 \pm 0.76^{\mathrm{b}, \mathrm{d}, \mathrm{e}}$ & $60.00 \pm 0.65^{a, b, c}$ & $56.40 \pm 1.50^{a, b, c}$ & 0.000 \\
\hline AST (U/L) & $178.85 \pm 2.85^{b}$ & $221.85 \pm 3.46^{\mathrm{a}, \mathrm{c}, \mathrm{d}, \mathrm{e}}$ & $182.50 \pm 7.64^{\mathrm{b}, \mathrm{e}}$ & $170.28 \pm 3.27^{b}$ & $166.00 \pm 1.64^{\mathrm{b}, \mathrm{c}}$ & 0.000 \\
\hline GGT (U/L) & $4.00 \pm 0.57^{\mathrm{b}}$ & $8.00 \pm 0.57^{\mathrm{a}, \mathrm{c}, \mathrm{d}, \mathrm{e}}$ & $5.16 \pm 0.87^{b}$ & $4.71 \pm 0.83^{\mathrm{b}}$ & $3.60 \pm 1.12^{\mathrm{b}}$ & 0.003 \\
\hline HDL (mg/dL) & $21.28 \pm 0.96^{\mathrm{b}}$ & $13.57 \pm 1.04^{\mathrm{a}, \mathrm{c}, \mathrm{d}, \mathrm{e}}$ & $19.33 \pm 0.76^{b}$ & $21.28 \pm 1.86^{\mathrm{b}}$ & $19.40 \pm 1.20^{\mathrm{b}}$ & 0.001 \\
\hline LDL (mg/dL) & $10.85 \pm 1.10^{\text {bee }}$ & $16.14 \pm 1.31^{\mathrm{a}, \mathrm{c}, \mathrm{d}}$ & $10.00 \pm 0.68^{\mathrm{b}, \mathrm{e}}$ & $10.57 \pm 2.40^{\mathrm{b}, \mathrm{e}}$ & $15.60 \pm 1.28^{\mathrm{a}, \mathrm{c}, \mathrm{d}}$ & 0.017 \\
\hline TG (mg/dL) & $57.00 \pm 2.29^{\mathrm{b}, \mathrm{e}}$ & $76.71 \pm 2.34^{\mathrm{a}, \mathrm{c}, \mathrm{d}, \mathrm{e}}$ & $55.50 \pm 3.54^{\mathrm{b}}$ & $51.28 \pm 2.25^{\mathrm{b}}$ & $48.00 \pm 4.74^{\mathrm{a}, \mathrm{b}}$ & 0.000 \\
\hline $\mathrm{CH}(\mathrm{mg} / \mathrm{dL})$ & $46.71 \pm 1.61^{\mathrm{b}, \mathrm{d}, \mathrm{e}}$ & $58.00 \pm 1.75^{\mathrm{a}, \mathrm{c}, \mathrm{d}, \mathrm{e}}$ & $40.83 \pm 1.97^{\mathrm{b}, \mathrm{d}, \mathrm{e}}$ & $29.85 \pm 2.87^{a, b, c}$ & $28.20 \pm 2.55^{\mathrm{a}, \mathrm{b}, \mathrm{c}}$ & 0.000 \\
\hline Insulin $(\mu \mathrm{U} / \mathrm{mL})$ & $0.15 \pm 0.02^{\mathrm{b}, \mathrm{d}, \mathrm{e}}$ & $0.09 \pm 0.01^{\mathrm{b}, \mathrm{d}, \mathrm{e}}$ & $0.13 \pm 0.01^{\mathrm{d}, \mathrm{e}}$ & $0.24 \pm 0.01^{\mathrm{a}, \mathrm{b}, \mathrm{c}, \mathrm{d}}$ & $0.34 \pm 0.01^{\mathrm{a}, \mathrm{b}, \mathrm{c}, \mathrm{d}}$ & 0.000 \\
\hline
\end{tabular}

Each group represents the mean \pm SEM for seven rats. ${ }^{a} \mathrm{p}<0.05$ vs control group; ${ }^{b} \mathrm{p}<0.02$ vs OLZ group; ${ }^{\mathrm{p}}<<0.03$ vs OLZ+TQ-1 group; ${ }^{\mathrm{d} p}<0.04$ vs OLZ+TQ-2 group; ${ }^{e} \mathrm{p}<0.05$ vs OLZ+TQ-3 group. OLZ - olanzapine; TQ - thymoquinone; ALT - alanine aminotransferase; AST - aspartate aminotransferase; GGT - gamma glutamyl transpeptidase; HDL - high-density lipoprotein cholesterol; LDL - low density lipoprotein cholesterol; TG - triglycerides; CH - total cholesterol; OLZ+TQ-1 - OLZ+25 mg/kg TQ; OLZ+TQ-2 - OLZ+50 mg/kg TQ; OLZ+TQ-3 - OLZ+100 mg/kg TQ. Amounts of $4 \mathrm{mg} / \mathrm{kg}$ once a day for the first week, $8 \mathrm{mg} / \mathrm{kg}$ once a day for the second week of OLZ were given to all groups, except for control group

Tab. 4. Effects of olanzapine and thymoquinone on apoptotic index $(\%)$ in rat liver.

\begin{tabular}{lc}
\hline Groups & Apoptotic Index (\%) $(\mathrm{AI} ;$ mean $\pm \mathrm{SD})$ \\
\hline Control & $2.60 \pm 0.89^{\text {b.c.d.e }}$ \\
OLZ & $17.60 \pm 1.14^{\text {a.c.d.e }}$ \\
OLZ+TQ-1 & $7.80 \pm 1.30^{\text {a.b }}$ \\
OLZ+TQ-2 & $7.40 \pm 1.14^{\text {a.b }}$ \\
OLZ+TQ-3 & $8.50 \pm 5.05^{\text {a.b }}$ \\
\hline
\end{tabular}

The apoptotic index of all the groups. Values are mean \pm SD for seven rats in each group. a - Significant from control; $\mathrm{b}-$ Significant from OLZ; $\mathrm{c}-$ Significant from OLZ+TQ-1; d - Significant from OLZ+TQ-2; e - Significant from OLZ+TQ-3 $(\mathrm{p} \leq 0.05)$. OLZ - olanzapine; TQ - thymoquinone; OLZ+TQ-1 - OLZ+25 $\mathrm{mg} / \mathrm{kg}$ TQ; OLZ+TQ-2 - OLZ+50 mg/kg TQ; OLZ+TQ-3-OLZ+100 mg/kg TQ. Amounts of $4 \mathrm{mg} / \mathrm{kg}$ once a day for the first week, $8 \mathrm{mg} / \mathrm{kg}$ once a day for the second week of OLZ were given to all groups, except for control group

Ameliorative effects of TQ treatment against OLZ administration significantly increased the serum TAS level and decreased TOS, OSI levels $(\mathrm{p}<0.05)$. The control group had significantly higher TAS level compared to both OLZ and OLZ+TQ-3 groups ( $p=0.000$ and $p=0.002$, respectively). The OLZ+TQ-1 group had significantly higher TAS level compared to control, OLZ, OLZ+TQ-2 and OLZ+TQ-3 groups ( $\mathrm{p}<0.001)$. The OLZ+TQ-2 group had significantly higher TAS level than both OLZ and OLZ+TQ-3 groups ( $\mathrm{p}=0.002$ and $\mathrm{p}=0.018$, respectively) (Tab. 2, Fig. 2). TOS level was significantly higher in OLZ group compared to control, OLZ+TQ-1, OLZ+TQ-2 and OLZ+TQ-3 groups $(\mathrm{p}<0.002)$. The OLZ+TQ-1 group had a significantly lower TOS level than both control and OLZ+TQ-3 group $(\mathrm{p}=0.019$ and $\mathrm{p}$ $=0.002$, respectively). The OLZ $+\mathrm{TQ}-3$ group had a significantly higher TOS level compared to OLZ+TQ-2 group $(\mathrm{p}<0.030)$ (Tab. 2, Fig. 2). OSI level was significantly higher in OLZ group compared to control, OLZ+TQ-1 and OLZ+TQ-2 groups ( $\mathrm{p}=0.003$, $\mathrm{p}=0.002$ and $\mathrm{p}=0.003$, respectively) (Tab. 2, Fig. 2).

ALT, AST, GGT, LDL, glucose, TG, and CH levels were significantly increased in the OLZ group compared to the control, OLZ+TQ-1, OLZ+TQ-2 and OLZ+TQ-3 groups, while the HDL and insulin levels were decreased $(\mathrm{p}<0.01)$. ALT and $\mathrm{CH}$ levels were significantly lower in the OLZ+TQ-2 group compared to the
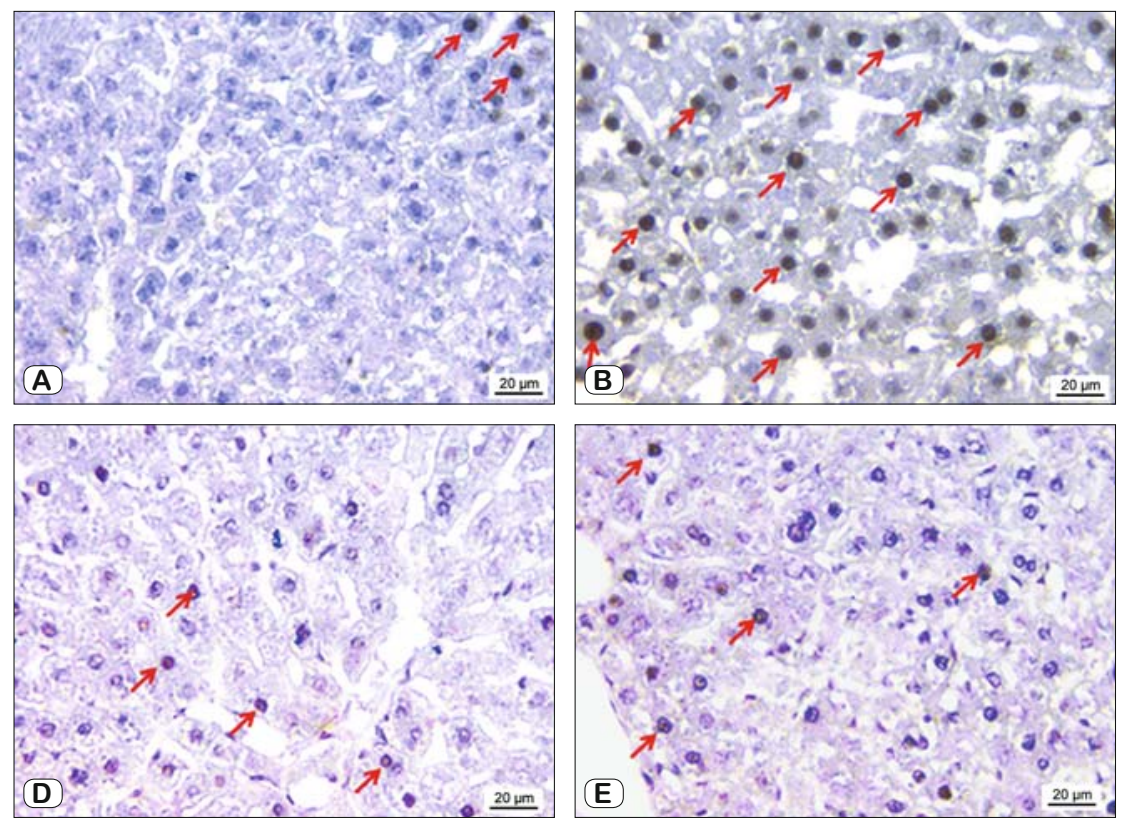

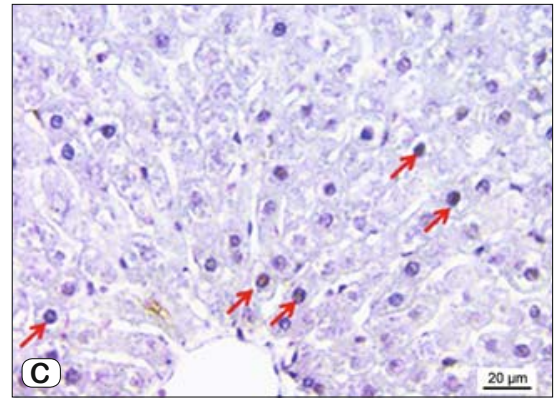

Fig. 4. Representative p hotomicrographs of Tunel staining in all five groups (scale bars $=\mathbf{2 0}$ $\mu \mathrm{m}$ ), showing: (A) Group 1 (control) only few Tunel-positive cells (arrow); (B) Group 2 (OLZ) a lot of Tunel-positive cells (arrows); (C) Group 3 (OLZ+TQ-1), (D) Group 4 (OLZ+TQ-2) and (E) Group 5 (OLZ+TQ-3) similarly rare Tunelpositive cells (arrows). 
OLZ+TQ-1 group $(\mathrm{p}<0.01)$. ALT, AST and CH levels were significantly lower in the OLZ+TQ-3 group compared to the OLZ+TQ-1 group, while the LDL level was increased $(\mathrm{p}<0.02)$. LDL level was significantly lower in the OLZ+TQ-2 group compared to the OLZ+TQ-3 group $(\mathrm{p}<0.03)$. ALT and CH levels were significantly lower in the OLZ+TQ-2 group compared to the control group ( $\mathrm{p}$ $<0.02$ ). ALT, TG and CH levels were significantly lower in the OLZ+TQ-3 group compared to the control group, while the LDL level was increased $(\mathrm{p}<0.05)$ (Tab. 3, Fig. 3).

\section{Evaluation of apoptosis in liver tissue}

The results of the apoptotic index are shown in Table 4 and Figure 4. Using TUNEL assay to detect apoptotic hepatic cells in the liver sections, the control (Fig. 4A) group showed only a few TUNEL-positive cells. The number of TUNEL-positive cells markedly increased in OLZ (Fig. 4B) group compared with the control group ( $\mathrm{p}<0.05$ ). OLZ+TQ-1 (Fig. 4C), OLZ+TQ-2 (Fig. 4D) and OLZ+TQ-3 (Fig. 4E) groups were similar and showed rare TUNELpositive cells. Treatment with TQ (OLZ+TQ-1, OLZ+TQ-2 and OLZ+TQ-3 groups) (Fig. 4C, 4D and 4E) reduced the number of TUNEL-positive cells as compared with the OLZ group $(\mathrm{p}<0.05)$.

\section{Discussion}

Many efforts have been ongoing for the identification and development of adjunctive medications to prevent the side effects induced by antipsychotics, including OLZ. Despite the advantages of treatment with OLZ, the reported side effects including, weight gain, impaired glucose metabolism and dyslipidemia, elevated liver enzymes and hepatotoxicity should be taken into account $(26,27)$. Therefore, it is extremely important to prevent adverse effects and other metabolic disorders induced by OLZ. In addition, its effects on the liver, the primary organ for drug activation and detoxification, still remains unclear. Dietary intake of natural compounds, including TQ, can inhibit metabolic adverse effects of OLZ and thereby may reduce the risk factors in the liver. Hence, the aim of the current study was to investigate the effect of TQ treatment against administration of OLZ in liver of rats $(28,29)$.

The increased energy storage induced by OLZ was extensively considered a result of both increased energy intake (such as hyperphagia) $(30,31)$ and reduced energy expenditure (such as decreased thermogenesis) (32-34). In a study performed by Ader et al showed that dogs treated with OLZ for 4-6 weeks and assessed parameters related to weight gain, adiposity and insulin resistance $(35,36)$. In the present study, initial and final body weight of experimental animals were measured. All doses of TQ caused dose-dependent decreases in body weight of the animals as compared to OLZ administrated rats. However, TQ co-treatment ameliorated these changes with a more obvious effect and significantly decreased only in group with $25 \mathrm{mg} / \mathrm{kg}$ dose. So far multiple in vivo studies have reported excellent effectiveness of TQ treatment on liver diseases (39). Hence TQ, a dietary supplement, significantly prevents OLZ-induced weight gain, which might suggest a potential effectiveness on human subjects. Therefore, TQ is a safe compound for co-administration with OLZ for mitigating antipsychotic-induced weight gain/obesity without influencing their therapeutic action. In addition, these results are certain to influence the choice of protective compound when several options exist and to institute preemptive strategies for weight management.

We investigated whether OLZ causes alterations in glucose metabolism and insulin secretion. Our findings confirmed that administration of OLZ caused a major impairment of glucose metabolism and a diminution of insulin action in liver. However, there are several other explanations possible. The explanation that fits best with the current data is that OLZ might be acting directly or indirectly to increase glucose level. Accordingly, a study conducted by Chintoh et al (2009) demonstrated that increased glucose was observed in OLZ-treated rats (37). On the other hand, in our study, TQ prevented the increase in fasting plasma glucose, impaired glucose tolerance and alteration in insulin sensitivity. Accordingly, the antihyperglycemic effect of TQ has been reported earlier in streptozotocin-nicotinamide-induced diabetic rats (38). The exact mechanisms of action of TQ against OLZ, impaired glucose tolerance and insulin resistance are not known. A further molecular study is required to find out the exact mechanisms of action of TQ against OLZ-induced impaired glucose tolerance and insulin resistance.

Our results indicated that OLZ exposure produced a significant increase in the activity of liver enzymes. ALT, AST and GGT indicating a damaged structural and functional hepatic integrity. Oral supplementation of TQ lowers the liver injury scores, restores the elevated serum ALT, AST and GGT activities. TQ co-treatment ameliorated these changes in all doses, especially the more obvious effect in high dose. These results are supported by literature data (40). In addition, we demonstrated that TQ prevented the increase in TG, total $\mathrm{CH}$ and LDL as well as decrease in HDL caused by OLZ consumption. All doses of TQ caused dose-dependent decreases in serum lipids as compared to OLZ administrated rats. However, TQ co-treatment ameliorated these changes with more obvious effect and significantly decreased only in group with 100 $\mathrm{mg} / \mathrm{kg}$ dose. The effect of TQ on serum lipids has been reported earlier in experimental conditions (20). This finding is probably a consequence of feeding behavior and increase in body weight. Although underlying physiological pathways are not fully understood, the present findings indicate that OLZ increases and TQ decreases the serum lipids.

Oxidative stress is one of the key mechanisms responsible for liver damage and disease progression. TAS measurement has been used to evaluate the overall performance of the antioxidant system. TOS measurement provides a sensitive index of lipid peroxidation and oxidative stress (41). The TOS/TAS ratio is termed as "OSI", which is an indicator of the oxidative stress degree (42). In the present study, we have observed that TQ could protect the liver from OLZ-induced liver injury. We measured TAS, TOS, and OSI levels at the same time to evaluate the oxidative stress status more accurately, and found that serum TAS levels increased and TOS/OSI levels prominently decreased with TQ treatment as in the previous studies (44). Likewise, OLZ administration resulted in a decrease in the liver TAS level and have also reported a decline in TAS after OLZ exposure (43). The beneficial effects of 
TQ, obtained in the current work, are very likely due to its strong antioxidant properties. The mechanisms of TQ antioxidant action are not yet clear. Nevertheless, TQ is known to reduce oxidative stress not only through the direct antioxidant effect but also indirectly. Previous studies reported that TQ acted as a strong reactive oxygen species (ROS) scavenger $(45,46)$.

Histopathological evaluation of liver showed severe damage ensued by loss of liver normal architecture which include vacuolar degeneration of hepatocytes and fatty changes in OLZ-administrated rats. These toxic effects were effectively prevented by antioxidant TQ administration. Among the 3 doses, $100 \mathrm{mg}$ of $\mathrm{TQ} / \mathrm{kg}$ body weight was found to provide optimum protective effect on liver against OLZ-induced abnormal changes. Histological observations added more evidence to the protective effect of TQ. The present study indicates that OLZ damaged the histological structure, impaired the function, inhibited the endogenous antioxidant defense system and stimulated TOS in the rat liver tissue. Besides, our results demonstrate, for the first time, that TQ oral supplementation, at safe doses, has a remarkable protective effect against OLZ-induced liver damage in rats. This protection makes TQ a promising agent in a variety of conditions where cellular damage is a consequence of oxidative stress. Therefore, OLZ-induced liver injury causes increased ROS formation and subsequent toxic events. Accordingly, in our study, TQ treatment of the cells against OLZ exposure had a great effect on apoptotic cell injury and death. The underlying protective mechanism of TQ may be associated with the suppression of apoptosis via death receptor-mediated pathways. Hence, it can be assumed that the antioxidant activity of TQ may be due to the effect on mitochondriaindependent apoptotic pathway which has to be supported with further experimentations. Therefore, TQ may be the best choice against OLZ-induced side effects.

On the one hand, co-treatment of OLZ-exposed rats with TQ in the present study significantly affected the liver OLZ burden. On the other hand, it improved the histopathological changes, ameliorated the impaired hepatic function, enhanced the reduced TAS, and inhibited the elevated TOS, and OSI levels in the liver. OLZ mediated ROS formation by diminishing antioxidant levels. Oxidative stress and antioxidant depletion could lead to apoptotic cell death (47). In this study, we found that TQ had a significant protective role in apoptotic cell death, which might be due to the ROS scavenging property. Taken the previous findings and suggestions together, it can be concluded that TQ could prevent OLZ-induced liver injury and histological perturbations through the enhancement antioxidant defense system, suppression of oxidative stress, and attenuation of apoptosis.

In conclusion, TQ may be a promising agent to improve OLZ adverse effects, oxidative stress, and apoptotic status, and so reduce weight gain and prevent liver damage in patients. Thus, daily consumption of TQ should be considered as a promising way to prevent liver damage. Our results could be used for planning strategies to protect against adverse effects of OLZ in the liver and also in other organs. Hence, further in vivo and clinical studies are needed to confirm the protective effects of TQ in patients receiving OLZ.

\section{References}

1. Han C, Wang SM, Kato M, Lee SJ, Patkar AA, Masand PS, Pae CU. Second generation antipsychotics in the treatment of major depressive disorder: current evidence. Expert Rev Neurother 2013; 13: 851-870.

2. Jacob R, Chowdhury AN. Metabolic comorbidity in schizophrenia. Indian J Med Sci 2008; 62 (1): 23-31.

3. Bear MF, Connors BW, Paradiso MA. Neurociências: desvendando o sistema nervoso. In: A regulação em longo prazo do comportamento alimentar. Artmed Editora, Porto Alegre 2002; 524-541.

4. Domínguez-Jiménez JL, Puente-Gutiérrez JJ, Pelado-García EM, Cuesta-Cubillas D, García-Moreno AM. Liver toxicity due to olanzapine. Rev Esp Enferm Dig 2012; 104: 617-618.

5. Taoka H, Hamamura T, Endo S, Miyata S, Toma K, Ishihara T, Kuroda S. Antipsychotics possessing antidepressive efficacy increase Golf protein in rat striatum. Psychopharmacology (Berlin) 2008; 201: 229-235.

6. Ali BH, Blunden G. Pharmacological and toxicological properties of Nigella sativa. Phytother Res 2003; 17: 299-305.

7. Rifaioglu MM, Nacar A, Yuksel R, Yonden Z, Karcioglu M, Zorba OU, Davarci I et al. Antioxidative and anti-inflammatory effect of thymoquinone in an acute Pseudomonas prostatitis rat model. Urol Int 2013; 91: 474-481.

8. Fararh KM, Shimizu Y, Shiina T, Nikami H, Ghanem MM, Takewaki T. Thymoquinone reduces hepatic glucose production in diabetic hamsters. Res Vet Sci 2005; 79 (3): 219-223.

9. Randhawa MA, Alghamdi MS, Maulik SK. The effect of thymoquinone, an active component of Nigella sativa, on isoproterenol induced myocardial injury. Pak J Pharm Sci 2013; 26 (6): 1215-1219.

10. Ashour AE, Abd-Allah AR, Korashy HM et al. Thymoquinone suppression of the human hepatocellular carcinoma cell growth involves inhibition of IL-8 expression, elevated levels of TRAIL receptors, oxidative stress and apoptosis. Mol Cell Biochem 2014; 389: 85-98.

11. Abdulhakeem Al-Maje A, Fadhel Al-Omar A, Mahmoud Nagi N. Neuroprotective effects of thymoquinone against transient forebrain ischemia in the rat hippocampus. European Journal of Pharmacology 2006; 543 (1-3): 40-47.

12. Fouda AM, Daba MH, Dahab GM. Sharaf El-Din OA. Thymoquinone ameliorates renal oxidative damage and proliferative response induced by mercuric chloride in rats. Basic and Clinical Pharmacology and Toxicology 2008; 103 (2): 109-118.

13. Su X, Ren Y, Yu N, Kong L, Kang J. Thymoquinone inhibits inflammation, neoangiogenesis and vascular remodeling in asthma mice. Int Immunopharmacol 2016; 38: 70-80.

14. Badary OA, Abd-Ellah MF, El-Mahdy MA, Salama SA, Hamada FM. Anticlastogenic activity of thymoquinone against benzo(a)pyrene in mice. Food and Chemical Toxicology 2007; 45 (1): 88-92.

15. Iskender B, Izgi K, Canatan H. Novel anti-cancer agent myrtucommulone-A and thymoquinone abrogate epithelial-mesenchymal transition in cancer cells mainly through the inhibition of PI3K/AKT signalling axis. Mol Cell Biochem 2016; 416: 71-84.

16. Badary OA, Al-Shabanah OA, Nagi MN, Al-Bekairi AM, Elmazar MMA. Acute and subchronic toxicity of thymoquinone in mice. Drug Develop Res 1998; 44: 56-61. 
17. Nili-Ahmadabadi A, Tavakoli F, Hasanzadeh G, Rahimi H, Sabzevari O. Protective effect of pretreatment with thymoquinone against aflatoxin B(1) induced liver toxicity in mice. Daru 2011; 19: 282-287.

18. Lupidi G, Scire A, Camaioni E, Khalife KH, De Sanctis G, Tanfani F, Damiani E. Thymoquinone, a potential therapeutic agent of Nigella sativa, binds to site I of human serum albumin. Phytomedicine 2010; 17: 714-720.

19. Albaugh VL, Henry CR, Bello NT, Hajnal A, Lynch SL, Halle B, Lynch CJ. Hormonal and metabolic effects of olanzapine and clozapine related to body weight in rodents. Obesity (Silver Spring) 2006; 14: 36-51.

20. Prabhakar P, Reeta KH, Maulik SK, Dinda AK, Gupta YK. Protective effect of thymoquinone against high-fructose diet-induced metabolic syndrome in rats. Eur J Nutr 2015; 54 (7): 1117-27.

21. Erel O. A novel automated method to measure total antioxidant response against potent free radical reactions. Clin Biochem 2004; 37: 112.

22. Erel O. A new automated colorimetric method for measuring total oxidant status. Clin Biochem 2005; 38: 1103.

23. Harma M, Harma M, Erel O. Oxidative stress in women with preeclampsia. Am J Obstet Gynecol 2005; 192: 656-657.

24. Tas $\mathbf{U}$, Ayan M, Sogut $\mathbf{E}$ et al. Protective effects of thymoquinone and melatonin on intestinal ischemia-reperfusion injury. Saudi J Gastroenterol 2015; 21: 284-289.

25. Can N, Catak O, Turgut B, Demir T, Ilhan N, Kuloglu T, Ozercan IH. Neuroprotective and antioxidant effects of ghrelin in an experimental glaucoma model. Drug Des Devel Ther 2015; 2(9): 2819-2829.

26. Deng C. Effects of antipsychotic medications on appetite weight and insülin resistance. Endocrinol Metab Clin North Am 2013; 42: 545-563.

27. Hasnain M, Vieweg WV, Fredrickson SK, Beatty-Brooks M, Fernandez A, Pandurangi AK. Clinical monitoring and management of the metabolic syndrome in patients receiving atypical antipsychotic medications. Primary Care Diabetes 2009; 3: 5-15.

28. Kahl R, Kappus H. Toxicology of the synthetic antioxidants BHA and BHT in comparison with the natural antioxidant vitamin E. Z Lebensm Unters Forsch 1993; 196: 329-338.

29. Pokorny J, Nguyen HTT, Korzack J. Antioxidant activities of rosemary and sage extracts in sunflower oil. Nahrung 1997; 41: 176-177.

30. Kluge M, Schuld A, Himmerich $\mathbf{H}$ et al. Clozapine and olanzapine are associated with food craving and binge eating: results from a randomized double-blind study. J Clin Psychopharmacol 2007; 27: 662-666.

31. Case M, Treuer T, Karagianis J, Hoffmann VP. The potential role of appetite in predicting weight changes during treatment with olanzapine. BMC Psychiatry 2010; 10: 72.

32. Kreuzer P, Landgrebe M, Wittmann $M$ et al. Hypothermia under olanzapine treatment: clinical case series and review of current literature. Nervenarzt 2012; 83: 630-637.

33. Kudoh A, Takase H, Takazawa T. Chronic treatment with antipsychotics enhances intraoperative core hypothermia. Anesth Analg 2004; 98: 111-115.
34. Davey KJ, O'Mahony SM, Schellekens H et al. Gender-dependent consequences of chronic olanzapine in the rat: effects on body weight, inflammatory, metabolic and microbiota parameters. Psychopharmacology (Berl) 2012; 221: 155-169.

35. Ader M, Kim SP, Catalano KJ et al. Metabolic dysregulation with atypical antipsychotics occurs in the absence of underlying disease. A placebo controlled study of olanzapine and risperidone in dogs. Diabetes 2005; 54: 862-871.

36. Cooper GD, Pickavance LC, Wilding JP, Harrold JA, Halford JC, Goudie AJ. Effects of olanzapine in male rats: enhanced adiposity in the absence of hyperphagia, weight gain or metabolic abnormalities. J Psychopharmacol 2007; 21 (4): 405-413.

37. Chintoh AF, Mann SW, Lam L, Giacca A, Fletcher P, Nobrega J, Remington G. Insulin resistance and secretion in vivo: effects of different antipsychotics in an animal model. Schizophr Res 2009; 108 (1-3): $127-133$.

38. Pari L, Sankaranarayanan C. Beneficial effects of thymoquinone on hepatic key enzymes in streptozotocin-nicotinamide induced diabetic rats. Life Sci 2009; 85 (23-26): 830-834.

39. Awad ASM, Abd Al Haleem EN, El-Bakly WM, Sherief MA. Thymoquinone alleviates nonalcoholic fatty liver disease in rats via suppression of oxidative stress, inflammation, apoptosis. Naunyn-Schmiedeberg's Arch Pharmacol 2016; 389: 381-391.

40. Galaly SR, Ahmed OM, Mahmoud AM. Thymoquinone and curcumin prevent gentamicin-induced liver injury by attenuating oxidative stress, inflammation and apoptosis. Journal of physiology and pharmacology 2014; 65 (6): 823-832.

41. Demirpence O, Sevim B, Yildirim M, Ayan NN, Mert D, Evliyaoglu O. Serum paraoxonase, TAS, TOS and ceruloplasmin in brucellosis. Int J Clin Exp Med 2014; 7: 1592.

42. Aycicek A, Ipek A. Maternal active or passive smoking causes oxidative stress in cord blood. Eur J Pediatr 2008; 167: 81.

43. Al-Chalabi BM, Thanoon IA, Ahmed FA. Potential effect of olanzapine on total antioxidant status and lipid peroxidation in schizophrenic patients. Neuropsychobiology 2009; 59 (1): 8-11.

44. Aydin MS, Kocarslan A, Kocarslan S et al. Thymoquinone protects end organs from abdominal aorta ischemia/reperfusion injury in a rat model. Rev Bras Cir Cardiovasc 2015; 30 (1): 77-83.

45. Mansour MA, Nagi MN, El-Khatib AS, Al-Bekairi AM. Effects of thymoquinone on antioxidant enzyme activities, lipid peroxidation and DT-diaphorase in different tissues of mice: a possible mechanism of action. Cell Biochem Funct 2002; 20: 143-151.

46. Badary OA, Taha RA, Abdel-Wahab MH. Thymoquinone is a potent superoxide anion scavenger. Drug Chem Toxicol 2003; 26: 87-98.

47. Armstrong JS, Jones DP. Glutathione depletion enforces the mitochondrial permeability transition and causes cell death in Bcl-2 overexpressing HL60 cells. Faseb J 2002; 16: 1263-1265.

Received May 1, 2017. Accepted May 25, 2017. 